\title{
Functional Roles of Netrin-1 in Osteoblast Differentiation
}

\author{
TSUYOSHI SATO ${ }^{1}$, SHOICHIRO KOKABU $^{1,2}$, YUICHIRO ENOKI $^{1}$, NAOKI HAYASHI $^{1}$, \\ MASAHITO MATSUMOTO $^{3}$, MITSUHIKO NAKAHIRA ${ }^{4}$, MASASHI SUGASAWA ${ }^{4}$ and TETSUYA YODA ${ }^{1}$ \\ ${ }^{1}$ Department of Oral and Maxillofacial Surgery, Saitama Medical University, Saitama, Japan; \\ ${ }^{2}$ Division of Molecular Signaling and Biochemistry, Department of Health Promotion, \\ Kyushu Dental University, Kitakyushu, Japan; \\ ${ }^{3}$ Division of Functional Genomics and Systems Medicine, \\ Research Center for Genomic Medicine, Saitama Medical University, Saitama, Japan; \\ ${ }^{4}$ Department of Head and Neck Surgery, Saitama Medical University International Medical Center, Saitama, Japan
}

\begin{abstract}
Aim: Recent studies have demonstrated that netrin1 plays a vital role in bone metabolism. Previous studies have shown that osteoblasts produce netrin-1 which affects osteoclast differentiation. However, the role of netrin-1 in osteoblast differentiation is not well understood. In this study, we explored the roles of netrin-1 in osteoblasts. Materials and Methods: Quantitative reverse-transcriptase polymerase chain reaction (qPCR), RNA interference for netrin receptors, the generation of netrin-1 plasmid, transfection of plasmids, and cell proliferation assay were performed. Results: During osteoblast differentiation by ascorbic acid, netrin-1 expression was significantly decreased. Gene expression related with osteoblast differentiation was down-regulated by netrin-1 treatment. We also found that osteoblast differentiation by bone morphogenetic protein-4 (BMP-4) was inhibited in the presence of recombinant netrin-1. Forced expression of both BMP-4 and netrin-1 significantly decreased alkaline phosphatase expression. On the other hand, Unc5b, neogenin, and $A 2 b$ which belong to netrin receptors were expressed by osteoblasts. Moreover, alkaline phosphatase expression was significantly decreased by knockdown for the combination of two receptors among these receptors. Conclusion: Netrin-1 is involved in the regulation of osteoblast differentiation.
\end{abstract}

Bone tissue is constantly being remodeled to maintain its homeostasis by osteoblasts and the resorption by osteoclasts

This article is freely accessible online.

Correspondence to: Tsuyoshi Sato, D.D.S., Ph.D., Department of Oral and Maxillofacial Surgery, Saitama Medical University, 38 Moro-hongou, Moroyama-machi, Iruma-gun, Saitama, 350-0495, Japan.Tel/Fax: +81 492761859, e-mail: tsato@ saitama-med.ac.jp

Key Words: Netrin-1, osteoblast, differentiation.
(1). The function of bone tissue is regulated by several factors which other organs produce to communicate with bones. Indeed, the vessels and nerves inside bone are essential for bone development and remodeling $(2,3)$. Previous studies have demonstrated that neural and vascular regulation factors, such as semaphorins and netrins, orchestrate bone metabolism. We have demonstrated that semaphorin $3 \mathrm{~A}$, which is a diffusible axonal chemorepellent that has an important role in axon guidance, regulates bone remodeling indirectly by modulating sensory nerve development and also found that it enhances the elongation of dendrite in osteocytes $(4,5)$. Semaphorin $3 \mathrm{~A}$ exerts an osteoprotective effect by both suppressing bone resorption and increasing bone formation (6).

Mammalian netrins including the secreted proteins (netrin1, netrin-3, netrin-4 and netrin-5) and membrane-bound proteins (netrin-G1 and netrin-G2) are involved in the regulation of both axon guidance and angiogenesis (7). The netrin system comprises of seven receptors including neogenin (Neo1), deleted in colorectal carcinoma (DCC), Unc5A, Unc5B, Unc5C, Unc5D, adenosine 2b (A2b), and Down syndrome cell adhesion molecule (DSCAM) (7). Among these netrins, netrin-1 (Ntn1) has involved in cytoskeleton reorganization and in epithelial cell adhesion and migration in lung, mammary gland, and pancreas (8-10). Recent studies showed that Ntn1 plays a crucial role in osteoclast differentiation $(11,12)$. As the function of osteoclasts is regulated by Ntn1, the question whether Ntn1 affects cellular function in osteoblasts has been raised. However, little is known about the role of Ntn 1 in osteoblasts. In this study, we explore whether Ntn1 affects the function of osteoblasts.

\section{Materials and Methods}

Cell culture, reagents and transfection. The mouse pre-osteoblastic cell line MC3T3-E1 cells which were kindly provided from Dr. Toru Ogasawara (Department of Oral and Maxillofacial Surgery, The University of Tokyo, Japan) were maintained in the medium, 
consisting of $\alpha$-modified minimum essential medium ( $\alpha$-MEM) (WAKO, Osaka, Japan) with 10\% fetal bovine serum (FBS) (BioWest, Nuaillé, France). For osteoblastic differentiation assay, cells were cultured in the medium containing ascorbic acid $(50 \mu \mathrm{g} / \mathrm{ml})$ and $\beta$-glycerophosphate $(10 \mathrm{mM})$. The medium was changed every 3 days. All cultures were maintained at $37^{\circ} \mathrm{C}$ in humidified air including $5 \% \mathrm{CO}_{2}$. Recombinant mouse Ntn1 (1109-N1-025) was purchased from R\&D systems (Minneapolis, MN, USA). Cells were transfected with plasmids using Lipofectamine 2000 (Thermo Scientific, Wilmington, DE, USA).

Cell proliferation assay. The cells were incubated with recombinant $\mathrm{Ntn} 1$ at the indicated concentration for 3 days. The sample cells were quantified using a Cell Titer 96 Aqueous One Solution Cell Proliferation Assay (Promega, Madison, WI, USA), according to the manufacturer's instructions. The measurements are represented by the means of at least three independent experiments, with each data point based upon six replicates.

Measurement of alkaline phosphatase (ALP) activity. Cells were plated at a density of $2 \times 10^{4}$ cells in 24 -well plates. After reaching confluence, cells were incubated with ascorbic acid $(50 \mu \mathrm{g} / \mathrm{ml})$ and $\beta$-glycerophosphate $(10 \mathrm{mM})$ in the presence or absence of recombinant Ntn1 at the indicated concentration for 7 days. ALP activity was assayed (WAKO, Tokyo, Japan) as previously described. The measurements are expressed as the means of three independent experiments, with each data point based on four replicates.

Quantitative reverse-transcriptase polymerase chain reaction ( $q P C R)$. To validate changes in gene expression, quantitative real time RTPCR (qPCR) analysis was performed using Applied Biosystems Prism 7900HT Sequence Detection System according to the manufacturer's instructions (Thermo Scientific, Wilmington, DE, USA) as previously described (13). Total RNA was extracted from cells using ISOGEN (Nippon Gene, Tokyo, Japan). We used SYBR Green-based detection. Reverse-transcriptase reaction was performed with High Capacity cDNA Reverse Transcription kit (Thermo Scientific, Wilmington, DE, USA). The cDNA was amplified by PCR using murine specific primers for alkaline phosphatase (Alp), bone gamma-carboxyglutamic acid-containing protein (Bglap), Ntn1, BMP-4, collagen 1 alpha (Col1a), DCC, Unc5a, Unc5b, Unc5c, Unc 5d, A2b, Neo1, DSCAM, and $\beta$-actin (Actb) as follows: Alp sense, 5'- GCTTTAAACCCAGACACAAG-3' and Alp antisense, 5'GCAGTAACCACAGTCAAGGT-3'; Bglap sense, 5'-TGCTTGTGA CGAGCTATCAG-3' and Bglap antisense, 5'-GAGGACAGGGAG GATCAAGT-3'; Ntn1 sense, 5'-GCAAGCTGAAGATGAACAGA3' and Ntn1 antisense, 5'-CTTTGTCGGCCTTCAGGA-3'; BMP-4 sense, 5'-GAGGAGTTTCCATCACGAAGA-3' and BMP-4 antisense, 5'-GCTCTGCCGAGGAGATCA-3'; Colla sense, 5'CATGTTCAGCTTTGTGGACCT-3' and Colla antisense, 5'-GCA GCTGACTTCAGGGATGT-3'; DCC sense, 5'-GCTTTTGTCTCA GCCAGGAC-3' and DCC antisense, 5'-CGCTCAAGTCATCC TGTTCA-3'; Unc5a sense, 5'-ACCTCTGAGGCTGAGGACTTC-3' and Unc5a antisense, 5'-GGCTTGTGCAGAGTGAGGTAG-3'; Unc5b sense, 5'-CCATGGACCGGTACCTAAACT-3' and Unc5b antisense, 5'-TGGCTACCAGCATCTCACTCT-3'; Unc5c sense, 5'AACTCTCTTGGGGGTCACCT-3' and Unc 5c antisense, 5'-TGCAG AGTGAGGATGACAGG-3'; Unc5d sense, 5'-CCTGAGTCCTGA AGTCACCTG-3' and Unc5d antisense, 5'-ATGTGGACTCATC CTCCACTG-3'; A2 $b$ sense, 5'-TCCCGCTCAGGTATAAAGGTT-3' and $A 2 b$ antisense, 5'-GGACACACCCAAAGAAGTTGA-3'; Neol sense, 5'-AGAGGGCATGAGTCAGAGGA-3' and Neol antisense, 5'-GCTGGAGTGGAAATGATGGT-3'; DSCAM sense, 5'-ATATCA GGCGCGAATAAACG-3' and DSCAM antisense, 5'-CCGTGATG TTCCTCATTGC-3'; Actb sense, 5'-AGAAGGACTCCTATGTGG GTGA-3' and Actb antisense, 5'-CATGATCTGGGTCAT CTTTTCA3'. SYBR green-based qPCR was performed using THUNDERBIRD SYBR qPCR Mix (TOYOBO, Osaka, Japan). Thermal cycling conditions were conducted as previously described (13). Values were normalized to $A c t b$ using the $2^{-\Delta \Delta C t}$ method. All samples were examined in triplicate assays.

Plasmids. Mouse Ntn1 (accession number NM_008744.2) and BMP4 (accession number NP_031580.2) was obtained by a standard RTPCR technique using PrimeSTAR HS DNA polymerase (TaKaRa, Ohtsu, Japan) and cloned into pcDNA3.1 expression vector (Thermo Scientific, Wilmington, DE, USA) as previously described (14).

Small interfering RNA (siRNA) transfection. RNA interference was performed as previously described (13). Stealth Select RNAi siRNA for murine Ntn1 (MSS207200, MSS207201, MSS207202) and Stealth RNAi Negative Control Duplex (Low GC, Medium GC) as si-control (si-CTL) were purchased from Thermo Scientific (Wilmington, DE, USA). Briefly, they were all transfected with Lipofectamine RNAi MAX (Thermo Scientific, Wilmington, DE, USA) according to the manufacturer's instructions. Results were representative of more than three individual experiments.

Statistical analysis. Comparisons between two groups were analyzed using Student's $t$-tests and comparisons among three groups were analyzed using One-Way Analysis of Variance and Bonferroni/Dunn methods $\left({ }^{\#} p<0.05,{ }^{\# \#} p<0.01\right)$. All values are represented as the mean \pm S.E.M. Results are representative examples of more than three independent sets of experiments.

\section{Results}

Ntn1 inhibits osteoblast differentiation in MC3T3-E1. First, we assessed Ntn 1 expression during osteoblast differentiation by qPCR analysis. As shown in Figure 1A, Ntn1 mRNA expression was decreased at 7 and 14 days after induction of ascorbic acid and $\beta$-glycerophosphate. To assess whether treatment of Ntn1 affects osteoblast differentiation, we added recombinant mouse $\mathrm{Ntn} 1$ to the culture of conditioned medium. collagen 1 alpha (Col1a), an early marker of osteogenic differentiation, was down-regulated in a dosedependent manner in the presence of Ntn1 (Figure 1B). Ntn1 inhibited ALP activity of MC3T3-E1 (Figure 1C). To confirm that the decrease of gene expression related with osteogenic differentiation is not due to the inhibition of cell proliferation, we assessed whether Ntn1 affects cell proliferation in MC3T3E1 cells. Ntn1 did not affect cell proliferation at all in MC3T3-E1 cells (Figure 1D).

To estimate the function of $\mathrm{Ntn} 1$ in osteogenic differentiation by induction of bone morphogenetic proteins (BMP), we overexpressed Ntn1 and/or BMP-4 in MC3T3-E1 cells (Figure 2A). Addition of recombinant Ntn1 significantly 


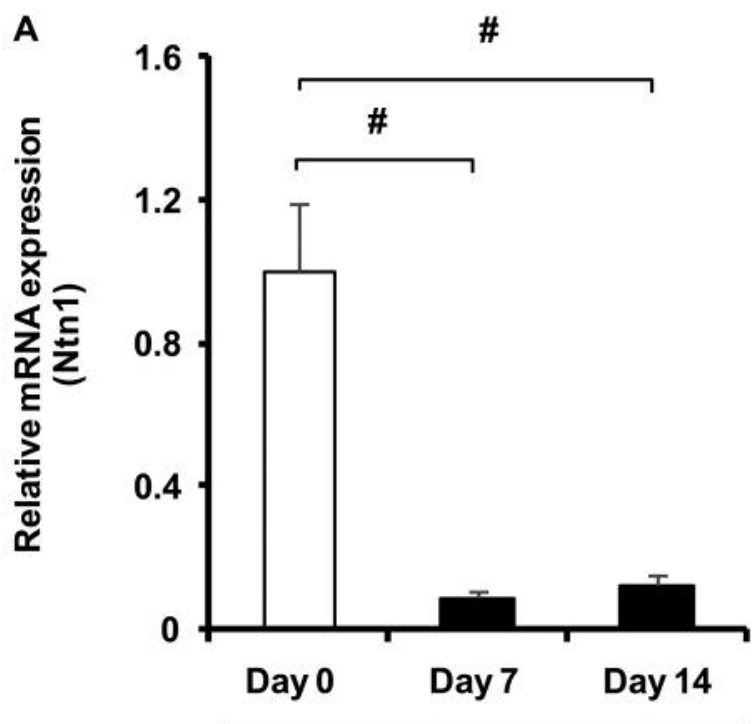

Conditioned medium

C

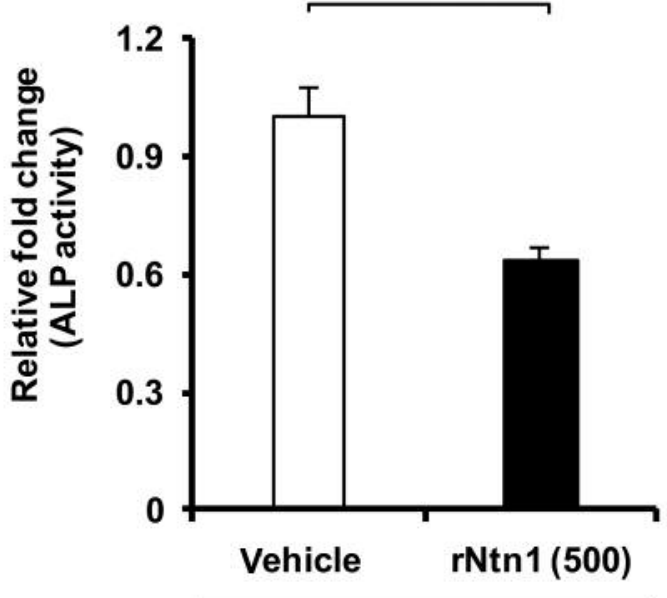

Conditioned medium

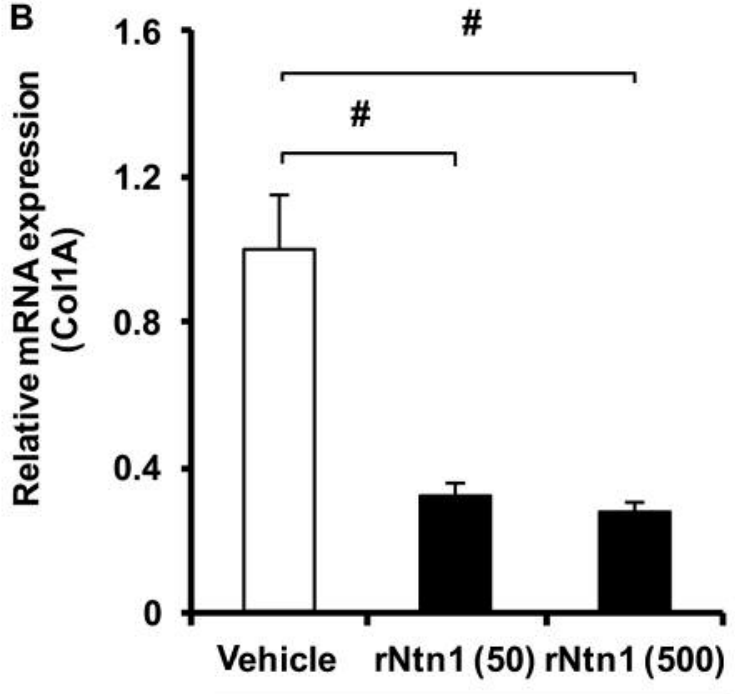

Conditioned medium

D

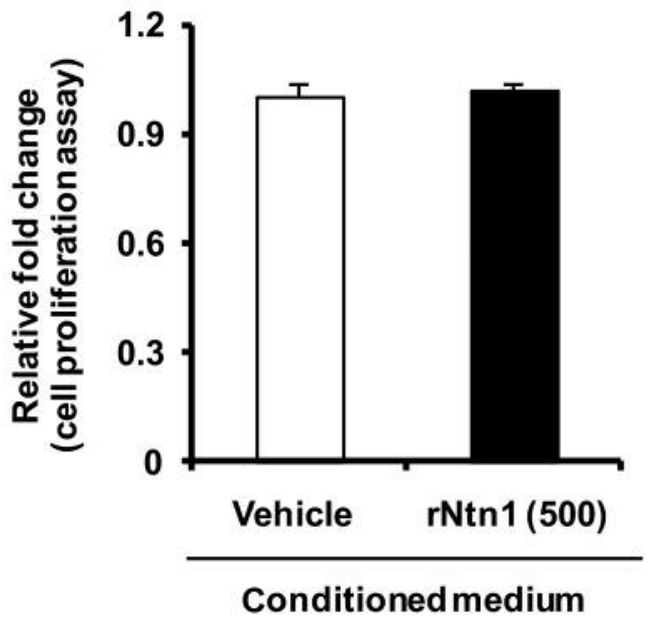

Figure 1. Ntn1 inhibits osteoblast differentiation. A. Expression of Ntn1 mRNA in MC3T3-E1 during osteoblast differentiation in the presence of ascorbic acid and $\beta$-glycerophosphate at 7 and 14 days estimated by qPCR analysis. B. Expression of Colla mRNA in MC3T3-E1 with recombinant Ntn1 (50 and $500 \mathrm{ng} / \mathrm{ml})$ for 3 days estimated by qPCR analysis. C. Effect of recombinant Ntn1 (500 $\mathrm{ng} / \mathrm{ml})$ on ALP activity of MC3T3-E1 at 7 days estimated by ALP activity assay. D. Effect of recombinant Ntn1 $(500 \mathrm{ng} / \mathrm{ml})$ on cell proliferation of osteoblastic cells at 3 days estimated by cell proliferation assay. Data are calculated from three repeated experiments. Recombinant Ntn1, rNtn1. ${ }^{p}<0.05$. Data are expressed as the means \pm S.E.M.

decreased Alp mRNA level in BMP-4 transfected MC3T3-E1 in a dose dependent manner (Figure 2B). Forced expression of Ntn1 significantly decreased both Alp and Col1a mRNA level (Figure 2C). Furthermore, simultaneous overexpression of both BMP-4 and Ntn1 also significantly inhibited osteoblast differentiation (Figure 2C). These results suggest that Ntn1 inhibits osteoblast differentiation.
Three netrin receptors are involved in the regulation of osteoblast differentiation by Ntn1. Next we assessed whether MC3T3-E1 cells express netrin receptors. As shown in Figure 3A-H, Neo1, A2b, and Unc5b were strikingly expressed by MC3T3-E1 compared to murine brain. Then we validated whether knockdown of these three receptors affect osteoblast differentiation in the presence of Ntn1. We confirmed that 

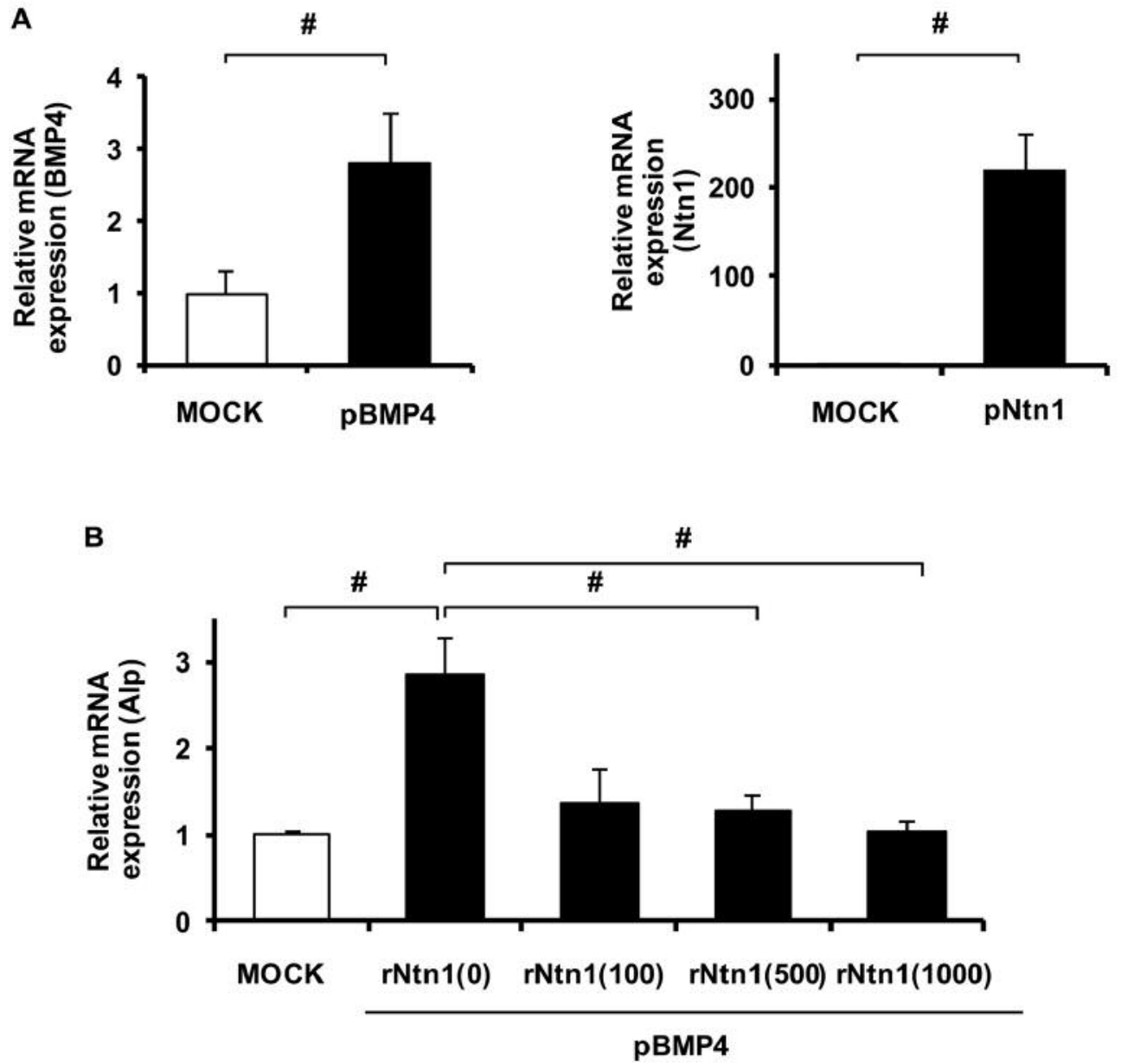

C
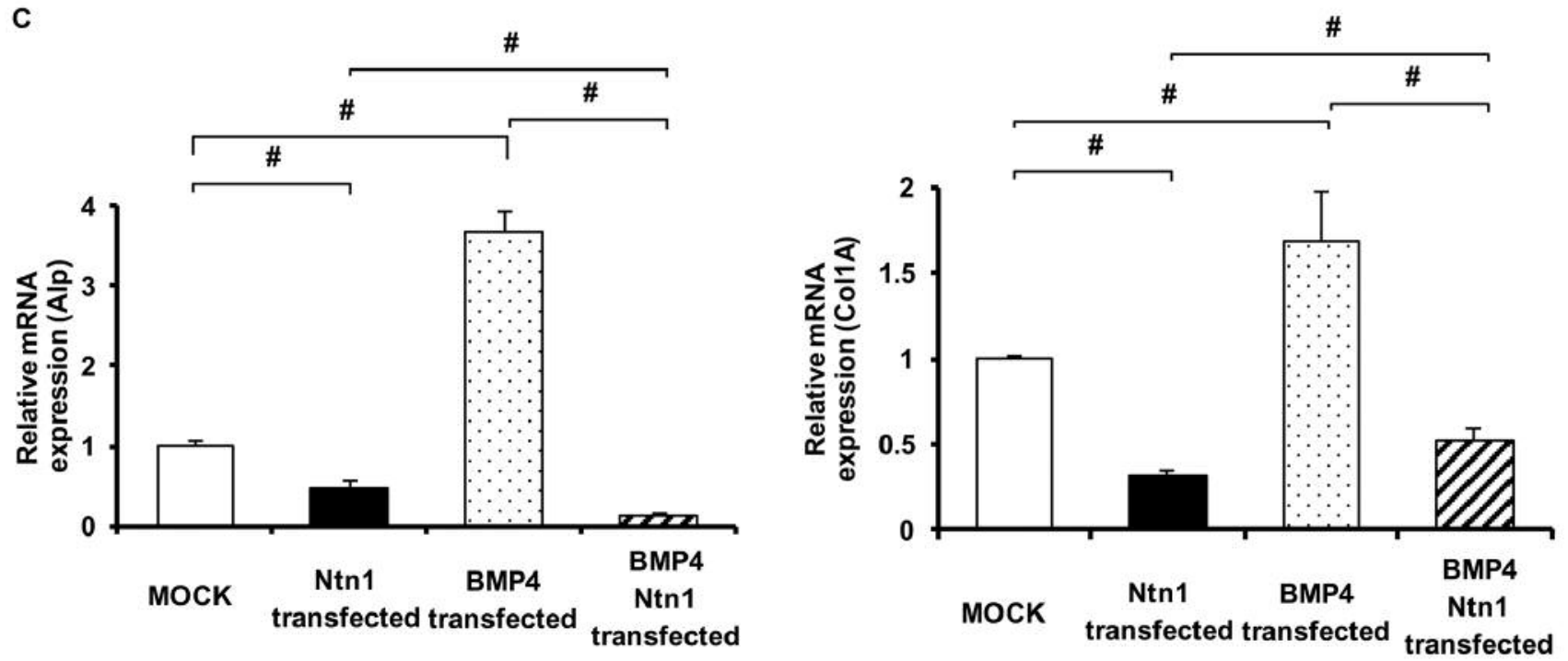

Figure 2. Ntn1 overexpression inhibits osteoblast differentiation. A. MC3T3-E1 cells were transfected with Ntn1 plasmid, BMP-4 plasmid, or an empty vector at 3 days estimated by qPCR analysis: (left panel) pBMP4, (right panel) pNtn4. B. Expression of Alp mRNA in BMP-4 transfected MC3T3-E1 with recombinant Ntn1 $(0,100,500$ and 1,000 $\mathrm{ng} / \mathrm{ml})$ for 3 days estimated by qPCR analysis. C. Expression of Alp and Colla mRNA in BMP-4 and/or Ntn1 transfected MC3T3-E1: (upper panel) Alp, (lower panel) Colla. Plasmid Ntn1, pNtn1; plasmid BMP-4, pBMP4; empty vector, MOCK; Recombinant Ntn1, rNtn1. ${ }^{\#} p<0.05$. Data are expressed as the means \pm S.E.M. 

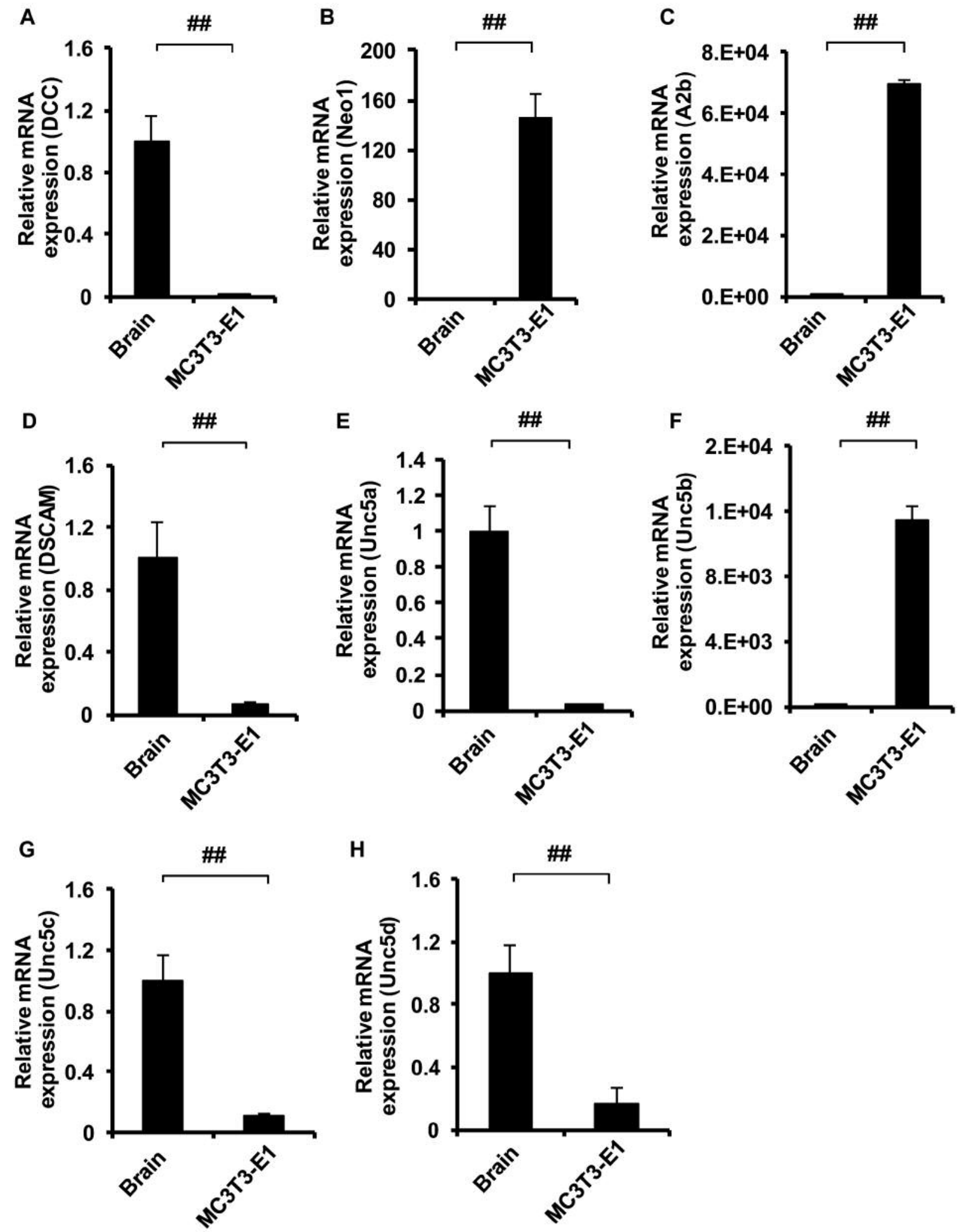

Figure 3. Netrin receptors $m R N A$ expression in MC3T3-E1. Expression of netrin receptors in MC3T3-E1 and murine brain estimated by qPCR analysis A. DCC mRNA. B. Neol mRNA. C. A2b mRNA. D. DSCAM mRNA. E. Unc5a mRNA. F. Unc5b mRNA. G. Unc5c mRNA. H. Unc5d mRNA. ${ }^{\#} p<0.05,{ }^{\#} p<0.01$. Data are expressed as the means \pm S.E.M. 
single knockdown of each receptor (i.e. siRNA for $\mathrm{A} 2 \mathrm{~b}$, Unc5b, Neo1) was successfully achieved (Figure 4A-C). Ntn1 inhibited Alp mRNA levels in siRNA for control transfected cells (Figure 4D). RNA interference of one receptor (A2b, Unc5b, Neo1) did not rescue the inhibition of differentiation by Ntn1 (Figure 4E-G). However, the combination of two among these three receptors recovers the inhibition of differentiation by Ntn1 (Figure 4H-J). These results suggest that the combination of two receptors is essential for regulating osteoblast differentiation by Ntn1.

\section{Discussion}

The present study demonstrated that Ntn1 plays a vital role for differentiation in osteoblasts. Osteoblast differentiation is partly controlled by bone morphogenetic protein (BMP) (15). Recent studies show that BMP is the key molecule to connect netrin with osteoblastic differentiation. Neogenin which belongs to one of the netrin receptors is involved in the regulation of BMPinduced Smad signaling and endochondral bone formation (16). Their studies provide evidence that neogenin may regulate chondrocyte maturation by promoting BMP induced BMP receptor association with lipid rafts, thus enhances effective BMP receptor concentration or BMP binding affinity and increases Smad phosphorylation and Runx 2 induction by using neogenin-deficient mice, suggesting that neogenin promotes chondrogenesis in vitro and in vivo. On the other hand, this controversial result has been already reported. Hagihara et al. reported that neogenin negatively regulates the functions of BMP and that this effect of neogenin is mediated by the activation of RhoA (17). However, it is conjectured that the inhibition of osteoblast differentiation by BMP via the activation of neogenin may not occur under the physiological condition.

Togari $e$ al. showed that Ntn1 is expressed by osteoblasts (18). Yagami et al. found that the expression of Ntn1 was decreased in the process whereby mesenchymal cells differentiate into osteoblasts by BMP (19). It has been reported that Ntn1 is involved in regulating bone remodeling. Mediero et al. demonstrated that Ntn1, which is produced by osteoclasts enhanced osteoclast differentiation by an autocrine/paracrine manner and Ntn1 deficient mice have markedly diminished osteoclasts, as well as increased cortical and trabecular bone density and volume compared to wildtype mice, suggesting that $\mathrm{Ntn} 1$ is a negative regulator in bone metabolism (11). In contrast, Maruyama et al. reported that Ntn1 suppresses osteoclast multinucleation, but not osteoclast differentiation and protected the mice against autoimmune bone destruction in vivo, indicating that $\mathrm{Ntn} 1$ is a positive regulator in bone metabolism (12). These researchers reported that Ntn1 does not affect osteoblast differentiation. As they use primary cells derived from murine calvaria which consist of a heterogeneous cell population, their results are different from our findings.
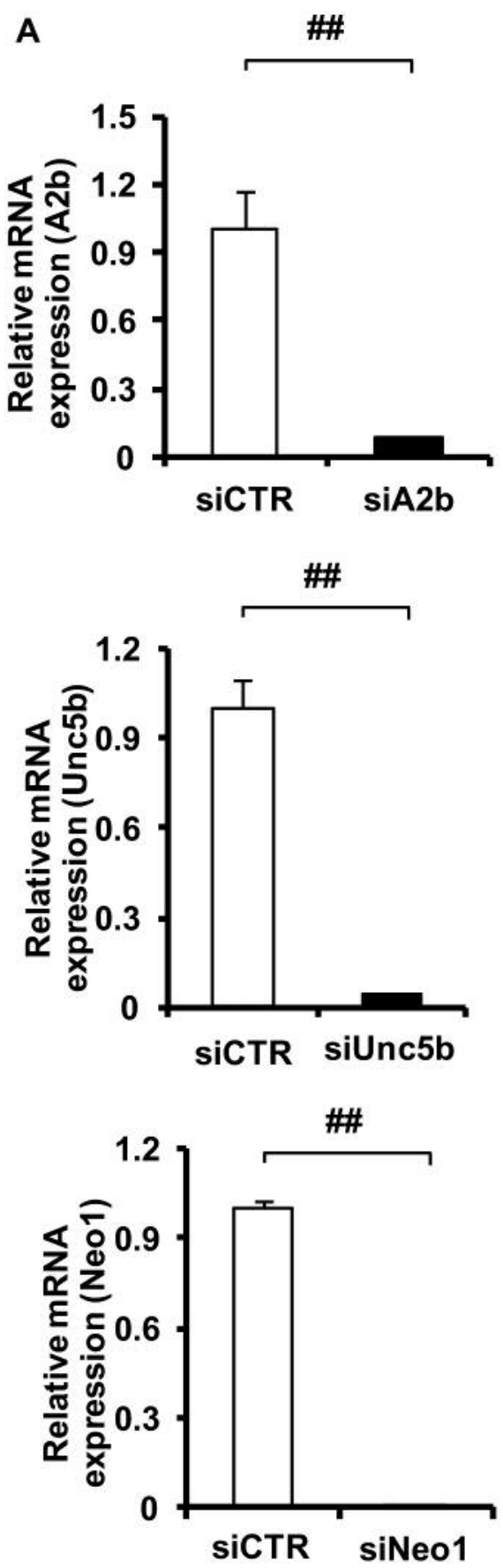

Figure 4. Continued

Ntn1 and its receptors are involved in regulating differentiation of various organs. Ntn1 is effective in regulating the expression of markers of neuroectodermal differentiation in human embryonal carcinoma cells (20). In mammary gland, Ntn1 facilitates functional differentiation of mammary epithelial cells (21). In terms of differentiation 

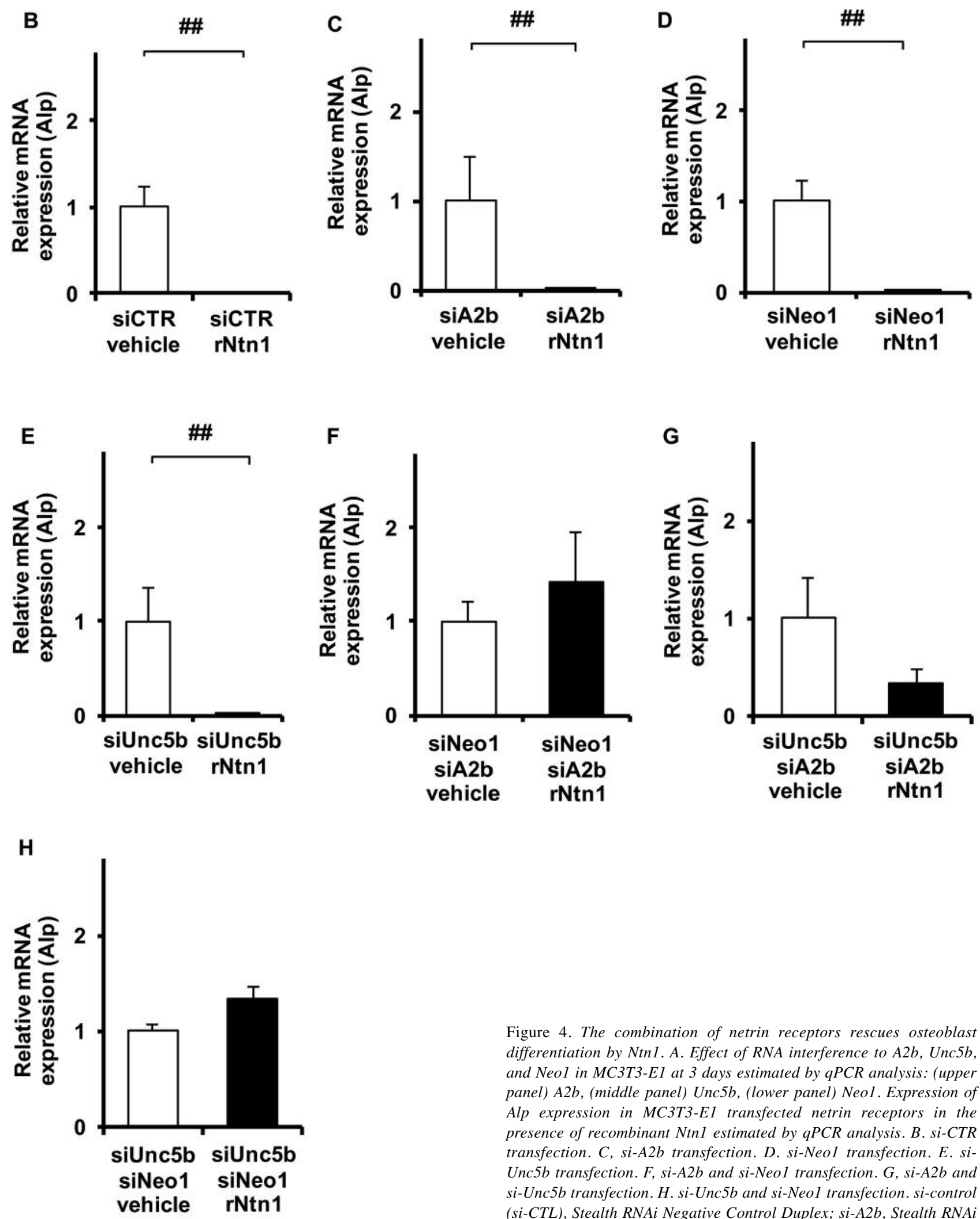

Figure 4. The combination of netrin receptors rescues osteoblast differentiation by Ntn1. A. Effect of RNA interference to A2b, Unc5b, and Neo1 in MC3T3-E1 at 3 days estimated by qPCR analysis: (upper panel) A2b, (middle panel) Unc5b, (lower panel) Neo1. Expression of Alp expression in MC3T3-E1 transfected netrin receptors in the presence of recombinant Ntn1 estimated by qPCR analysis. B. si-CTR transfection. C, si-A2b transfection. D. si-Neol transfection. E. siUnc $5 b$ transfection. F, si-A2b and si-Neo1 transfection. $G, s i-A 2 b$ and si-Unc5b transfection. H. si-Unc5b and si-Neol transfection. si-control (si-CTL), Stealth RNAi Negative Control Duplex; si-A2b, Stealth RNAi to A2b; si-Neo1, Stealth RNAi toNeo1; si-Unc5b, Stealth RNAi to Unc5b ${ }^{\#} p<0.05,{ }^{\# \#} p<0.01$. Data are expressed as the means \pm S.E.M. 
potential, Ozmadenci et al. showed that murine induced pluripotent stem cells which derived with recombinant Ntn1 turn off the endogenous Oct4/GFP promoter activity (22). Neogenin overexpression favors inner cell mass differentiation (23). In placenta, a discrete pattern of distribution of Ntn1 and its two receptors, Unc5B and DCC were observed (24). Moreover, $\mathrm{Xu}$ et al. demonstrated that negative regulation of $\mathrm{N}-\alpha$-acetyltransferase 10 towards $\mathrm{Ntn} 1$ and its receptor Unc5B were also detected upon treatment of retinoid acid that is used to induce morphological differentiation (25).

We have previously demonstrated that Ntn4, derived from vascular endothelial cells inhibits osteoclast differentiation (13). We speculate that netrin family may play a vital role for bone metabolism. Further investigations are required to clarify the role of netrins in bone metabolism.

In conclusion, we found that Ntn1 inhibits osteoblast differentiation and the combination of two netrin receptors is essential for regulating osteoblast differentiation by Ntn 1 .

\section{Acknowledgements}

This work was supported, in part, by a Grant-in-Aid (15K20556 to Yuichiro Enoki; 26462622 to Mitsuhiko Nakahira) from the Ministry of Education, Culture, Sports, Science, and Technology of Japan.

\section{References}

1 Delaisse JM: The reversal phase of the bone-remodeling cycle: cellular prerequisites for coupling resorption and formation. Bonekey Rep 3: 561, 2014.

2 Maes C: Role and regulation of vascularization processes in endochondral bones. Calcif Tissue Int 92: 307-323, 2013.

3 Houweling P, Kulkarni RN and Baldock PA: Neuronal control of bone and muscle. Bone 80: 95-100, 2015.

4 Fukuda T, Takeda S, Xu R, Ochi H, Sunamura S, Sato T, Shibata S, Yoshida Y, Gu Z, Kimura A, Ma C, Xu C, Bando W, Fujita K, Shinomiya K, Hirai T, Asou Y, Enomoto M, Okano H, Okawa A and Itoh $\mathrm{H}$ : Sema3 A regulates bone-mass accrual through sensory innervations. Nature 497: 490-493, 2013.

5 Niimura M, Sato T, Enoki Y, Okubo M, Kokabu S, Takeda S and Yoda T: Semaphorin 3A promotes dendrite elongation of osteocytes in association with down-regulation of CDK6. In Vivo 30: 231-236, 2016.

6 Hayashi M, Nakashima T, Taniguchi M, Kodama T, Kumanogoh A and Takayanagi H: Osteoprotection by semaphorin 3A. Nature 485: 69-74, 2012.

7 Rajasekharan S and Kennedy TE: The netrin protein family. Genome Biol 10: 239, 2009.

8 Dun XP and Parkinson DB: Role of Netrin-1 Signaling in Nerve Regeneration. Int J Mol Sci 18: 3, 2017.

9 Chen $\mathrm{H}$, Chen Q and Luo Q: Expression of netrin-1 by hypoxia contributes to the invasion and migration of prostate carcinoma cells by regulating YAP activity. Exp Cell Res 349: 302-309, 2016.

10 Liu C, Ke X, Wang Y, Feng X, Li Q, Zhang Y, Zhu J and Li Q: The level of netrin-1 is decreased in newly diagnosed type 2 diabetes mellitus patients. BMC Endocr Disord 16: 33, 2016.

11 Mediero A, Ramkhelawon B, Perez-Aso M, Moore KJ and Cronstein BN: Netrin-1 is a critical autocrine/paracrine factor for osteoclast differentiation. J Bone Miner Res 30: 837-854, 2015.
12 Maruyama K, Kawasaki T, Hamaguchi M, Hashimoto M, Furu M, Ito H, Fujii T, Takemura N, Karuppuchamy T, Kondo T, Fukasaka M, Misawa T, Saitoh T, Suzuki Y, Martino MM, Kumagai Y and Akira S: Bone-protective Functions of Netrin 1 Protein. J Biol Chem 291: 23854-23868, 2016.

13 Enoki Y, Sato T, Tanaka S, Iwata T, Usui M, Takeda S, Kokabu S, Matsumoto M, Okubo M, Nakashima K, Yamato M, Okano T, Fukuda T, Chida D, Imai Y, Yasuda H, Nishihara T, Akita M, Oda H, Okazaki Y, Suda T and Yoda T: Netrin-4 derived from murine vascular endothelial cells inhibits osteoclast differentiation in vitro and prevents bone loss in vivo. FEBS Lett 588: 2262-2269, 2014.

14 Kokabu S, Nguyen T, Ohte S, Sato T, Katagiri T, Yoda T and Rosen V: TLE3, transducing-like enhancer of split 3, suppresses osteoblast differentiation of bone marrow stromal cells. Biochem Biophys Res Commun 438: 205-210, 2013.

$15 \mathrm{Wu}$ M, Chen G and Li YP: TGF- $\beta$ and BMP signaling in osteoblast, skeletal development, and bone formation, homeostasis and disease. Bone Res 26: 16009, 2016.

16 Zhou Z, Xie J, Lee D, Liu Y, Jung J, Zhou L, Xiong S, Mei L and Xiong WC: Neogenin regulation of BMP-induced canonical Smad signaling and endochondral bone formation. Dev Cell 19: 90-102, 2010.

17 Hagihara M, Endo M, Hata K, Higuchi C, Takaoka K, Yoshikawa $\mathrm{H}$ and Yamashita T: Neogenin, a receptor for bone morphogenetic proteins. J Biol Chem 286: 5157-5165, 2011.

18 Togari A, Mogi M, Arai M, Yamamoto S and Koshihara Y: Expression of mRNA for axon guidance molecules, such as semaphorin-III, netrins and neurotrophins, in human osteoblasts and osteoclasts. Brain Res 878: 204-209, 2000.

19 Yagami K, Nakamura M, Nakamura H, Maki S, Yagasaki T and Udagawa N: Neural-inducing Factor Netrin-1 is Regulated in Chondrogenesis and Osteogenesis by BMP or Noggin. J Hard Tissue Biol 21: 141-150, 2012.

20 Mancino M, Esposito C, Watanabe K, Nagaoka T, Gonzales M, Bianco C, Normanno N, Salomon DS and Strizzi L: Neuronal guidance protein Netrin-1 induces differentiation in human embryonal carcinoma cells. Cancer Res 69: 1717-1721, 2009.

21 Strizzi L, Mancino M, Bianco C, Raafat A, Gonzales M, Booth BW, Watanabe K, Nagaoka T, Mack DL, Howard B, Callahan R, Smith GH and Salomon DS: Netrin-1 can affect morphogenesis and differentiation of the mouse mammary gland. J Cell Physiol 216: 824-834, 2008.

22 Ozmadenci D, Féraud O, Markossian S, Kress E, Ducarouge B, Gibert B, Ge J, Durand I, Gadot N, Plateroti M, BennaceurGriscelli A, Scoazec JY, Gil J, Deng H, Bernet A, Mehlen P and Lavial F: Netrin-1 regulates somatic cell reprogramming and pluripotency maintenance. Nat Commun 6: 7398, 2015.

23 Lee JH, Choi SS, Kim HW, Xiong WC, Min CK and Lee SJ: Neogenin as a receptor for early cell fate determination in preimplantation mouse embryos. PLoS One 9: e101989, 2014.

24 Dakouane-Giudicelli M, Alfaidy N and de Mazancourt P: Netrins and their roles in placental angiogenesis. Biomed Res Int 2014: 901941, 2014.

$25 \mathrm{Xu} \mathrm{H}$, Han Y, Liu B and Li R: Unc-5 homolog B (UNC5B) is one of the key downstream targets of $\mathrm{N}-\alpha$-Acetyltransferase 10 (Na10). Sci Rep 6: 38508, 2016.

Received January 18, 2017 Revised March 9, 2017 Accepted March 10, 2017 